\title{
FREQUENCY AND PREVENTION OF VERRUCA PLANTARIS IN ATHLETES TRAINING IN FITNESS MORE THAN 5 YEARS
}

\author{
Valentin Valtchev \\ National Sports Academy "Vassil Levski", Sofia, Bulgaria
}

\begin{abstract}
Introduction: Verruca Plantaris is a dermatovirosis, which occurs with single or multiple papulo-tumorous and papillomothous surfaces on the athletes' feet.

Aim: The aim of this study was to determine the prevalence of verruca plantaris in athletes training in fitness more than 5 years

Methodology: In our study there were included 70 randomly selected men athletes training in fitness more than 5 years. They were ranged by age from 25 to 30 years. Each of the study participants were examined by a dermatologist.

Results: Verruca Plantaris was found in 33 (47\%) of the tested athletes. Results of our study showed that at 10 of the athletes Verruca Plantaris affects only one of the legs and at 23 affects both feet. $72.7 \%(n=24)$ of the athletes were not able to train in fitness for at least 2 weeks during the acute phase of the Verruca Plantaris, which cause a disturbing of athletes' quality of life and distress.

Discussion and conclusions: Verruca Plantaris is caused by human papillomavirus and affects the fingers of the lower limbs and/or the feet. Verruca Plantaris could be extremely painful when walking. Verruca Plantaris affects the quality of life of athletes and reduces athletic performance. It is important to make a program for the prevention of the infection of Verruca Plantaris and to be applied in fitness.
\end{abstract}

Key words: Verruca Plantaris, athletes, fitness

INTRODUCTION

Warts (Verruca Plantaris) are a benign growth of the epithelium of the skin. (Bacelieri, Johnson, 2005; Beers, 2005; Bhat, 2001; Chouhy et al., 2013; Egawa et al., 1998).
Plantar warts are dermatovirosis that occurs with single or multiple papulo-tumorous formations with papillomatoses surface on the feet of athletes. (Bacelieri, Johnson, 2005; Beers, 2005; Bhat, 2001) (Figure 1)

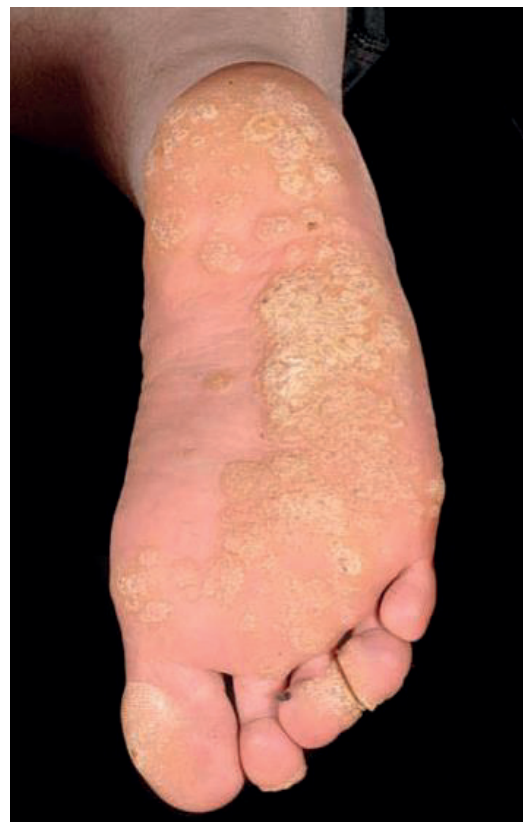

Figure 1. Mosaic warts on the sole (Kang, S., 2019. Fitzpatrick's Dermatology) 
Warts are caused by human papillomavirus (HPV). Warts are spread by direct contact from person to person or by contact with contaminated surfaces (flip flops, towels, room floors).

HPV is usually found in hot and humid places, such as changing rooms, public showers, and around swimming pools. Walking barefoot and bathing barefoot in public baths after a workout is a risk factor for HPV contamination. Micro traumas or skin lesions after intense training in the gym are the front door to HPV entry. (Bacelieri, Johnson, 2005; Beers, 2005; Bhat, 2001)

The incubation period for the viral warts occurrence is of varying duration and may be difficult to specify, as a number of factors affect its duration, such as the general health of the infected, presence of underlying diseases, immune status, infection intensity, etc. The incubation period may vary from a few weeks to several years.

Plantar warts may be very painful when walking, especially if they are located at a site of continuous pressure, i.e. the feet. (Chouhy et al., 2013; Egawa et al., 1998; Ferri, 2017; James, 2006)

Warts are most commonly seen in young people under 30 years of age. (Bacelieri, Johnson, 2005; Beers, 2005; Bhat, 2001; Chouhy et al., 2013; Egawa et al., 1998). There are two types of warts: single and mosaic type. Single or the so-called sol- itary warts may increase in size and eventually form satellite warts. The mosaic type of warts are clusters of small warts that grow close to each other. (Johnson, 1995; Kunnamo, 2005)

\section{METHODOLOGY}

The objective of our study was to investigate the frequency of Verruca Plantaris in athletes practicing fitness for more than 5 years.

The study was conducted in 70 randomly selected men who had been actively practicing fitness for more than 5 years. Sports gyms are randomly selected, among them there are big fitness and smaller, neighborhood gyms. All participants were between 25 and 30 years of age. In order to diagnose and localize Verruca Plantaris, all study participants were examined by a dermatologist. Athletes at whom we found warts were asked to complete a self-assessment questionnaire assessing their ability to train and the impact on their quality of life.

The questionnaire contains 10 demographic questions, a family history, a recurrence of the problem and the origin of the disease. Statistical analysis was performed with SPSS 21 and Variance analysis was made.

\section{RESULTS AND ANALISYS}

Verruca Plantaris was diagnosed in $47 \%(n=33)$ of the surveyed athletes. (Figure 2)

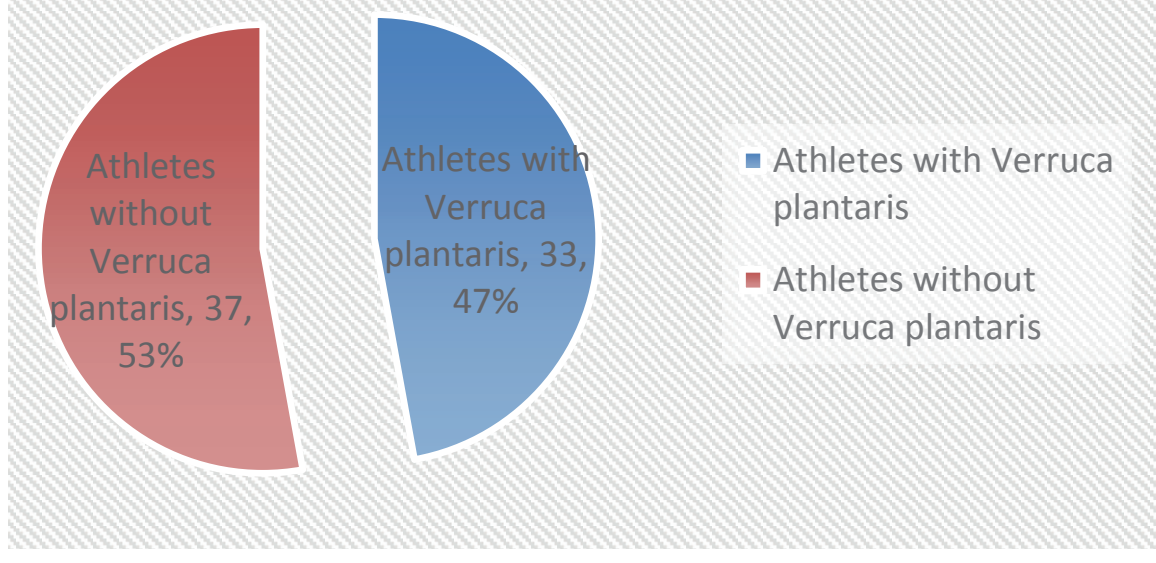

Figure 2. Athletes researched for Verruca Plantaris

Distribution in the Verruca Plantaris group accor- athletes Verruca Plantaris affected both legs. (Figding to the location was as follows: in 10 athletes ure 3).

Verruca Plantaris affected only one leg, and in 23 


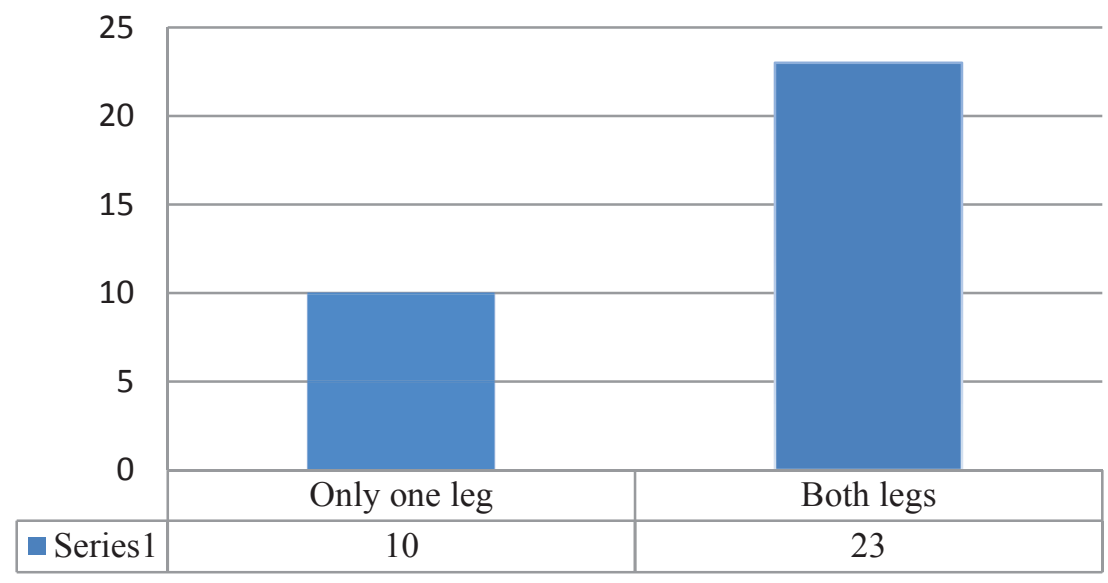

Figure 3. Localization of Verruca Plantaris

Very big percent of the athletes with warts, $72.7 \% \quad 55 \%$ of all respondents answered positively, that the $(n=24)$ were not able to work out in the gym for same skin problem occurred in some of their blood at least 2 weeks during the acute phase of Verruca relatives before. $57 \%$ of all athletes $(n=19)$ diagPlantaris, which affected their quality of life causing nosed with the disease claim that it recurs in time distress. (Figure 4).

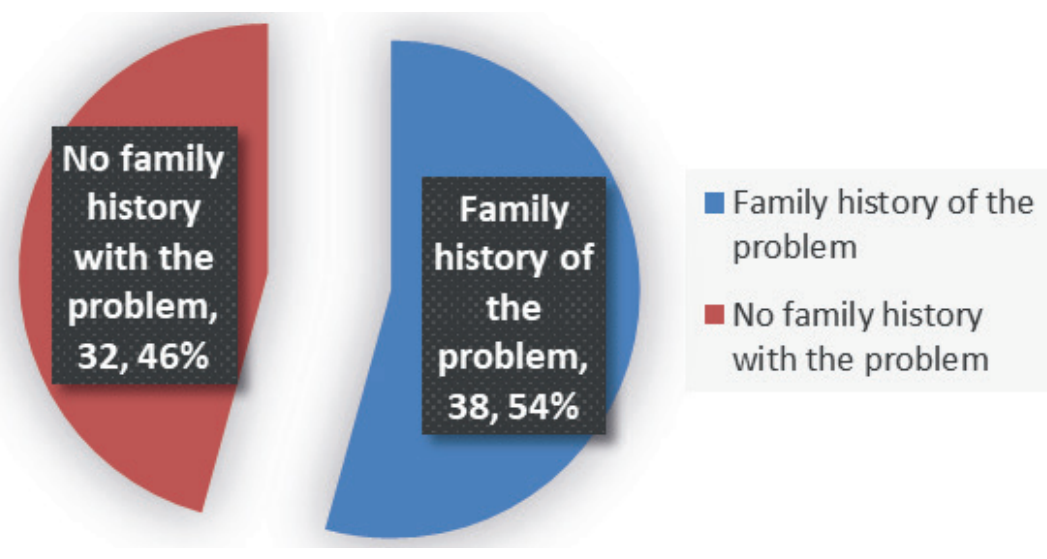

Figure 4. Occurrence of the problem in a blood relative Studying the origin of the disease in these athletes, $(n=44)$ say they have used someone else's fitness we found that $72 \%$ have walked barefoot in the equipment, such as a towel, flip flops, sports equlocker/dressing room, in the showers or inside ipment. (Figure 5.) the gym. Nearly $63 \%$ percent of those surveyed



Figure 5. Use of someone else's sports equipment in the hall (flip flops, towels, sports shoes) 
Also, we should not be underestimated the fact that different medications and supplements are used and some of them makes the problem to appear and some of them deal with the wart problem.

We asked our athletes who have warts whether they used corticosteroid medications to deal with the problem and $35 \%$ responded positively. We also asked if the athletes tested had ever used anabolic steroids and a fairly high percentage - $42 \%$ responded positively.

\section{DISCUSSION AND CONCLUSIONS}

Verruca Plantaris is caused by Human papilloma virus and affects the toes of the lower extremities or the feet. The clinical manifestation of Verruca Plantaris may lead to severe pain, especially when walking. Verruca Plantaris affects the quality of life of athletes while reducing their athletic performance. The use of public baths in the gyms without flip flops, or using someone else's equipment increases the risk of plantar wart contamination. The increased incidence of plantar warts found in athletes who had been exercising for more than 5 years was determined by the daily use of shared baths after each workout in the gym. We believe that there is a link between the appearance of plantar warts and the use of anabolic agents, as it is a well-known fact that they have the ability to lower the immune system and, accordingly, the immune response to infection is weaker. We did not find a connection between the size of the gyms. The problem is whether it is a large or small gym. This is probably due to the fact that cleanliness in the gym and bathing and changing rooms is important, not the number of visitors. Therefore, it is important to develop and implement a program aimed at preventing Verruca Plantaris in the fitness rooms. We believe that informing the visitors of the hall about this problem would prevent it from occurring as many of the sports hall and fitness's users are not aware of the reasons for its occurrence. The same goes for the owners of these gyms. Maintaining hygiene in gyms is crucial.

\section{REFERENCES}

Bacelieri, R., Johnson, SM. (2005). Cutaneous warts: An evidence-based approach to therapy. American Family Physician. 72 (4) pp. 647-652.

Beers, M. (2003). Merck Manual of Medical Information, 2nd home edition, Pocket Books.

Bhat, R., Vidya, K., Kamath, G. (2001). Topical formic acid puncture technique for the treatment of common warts. International Journal of Dermatology.40(6): 415-9 Chouhy, D. et al. (2013). Identification of human papillomavirus type 156, the prototype of a new human gammapapillomavirus species, by a generic and highly sensitive PCR strategy for long DNA fragments. J. Gen. Virol. 94 (Pt 3): 524-33

Egawa, K. et al. (1998). Human papillomavirus 57 identified in a plantar epidermoid cyst. Br. J. Dermatol. 138 (3): 510-4.

Ferri, F. (2017). Ferri's Clinical Advisor 2018 E-Book: 5 Books in 1, Elsevier Health Sciences. pp. 1375

James, D. et al. (2006). Andrews' Diseases of the Skin: clinical Dermatology. Saunders Elsevier. pp. 405

Johnson, L. (1995). Communal showers and the risk of plantar warts. J Fam Pract, vol. 40. pp. 136-8.

Kang, S. (2019). Fitzpatrick's Dermatology, Ninth Edition, 2-Volume Set, pp.3098

Kunnamo, I. (2005). Evidence-based Medicine Guidelines. John Wiley and Sons. p. 422.

\section{Corresponding address:}

Dr. Valentin Valtchev, PhD

Department of Physiology and Biochemistry, National Sports Academy "Vassil Levski"

21 Acad. Stephan Mladenov str., Stud.grad 1700 Sofia, Bulgaria E-mail: drviv1@abv.bg 